Tendenzen des Nachhaltigkeits-Research in Europa

\section{Vom Anbieterwachstum zum Qualitätsfortschritt?}

\author{
Nachhaltige Kapitalanlagen verzeichnen trotz des schwachen Marktumfeldes \\ weiterhin Zuwächse. Damit rückt auch das Nachhaltigkeits-Research stärker in \\ den Vordergrund. Denn die Analysen und Bewertungen von Wertpapieren nach \\ ökologischen und sozialen Kriterien bestimmen maßgeblich über die Ausgestal- \\ tung der nachhaltigen Investmentprodukte. Welche Anforderungen sollte eine \\ Research-Agentur erfüllen und wer sind die wichtigsten Akteure im euro- \\ päischen Raum?
}

$\mathrm{W}$

Von Marnie Bammert men aus einer Fülle von Angeboten wählen. Im deutschsprachigen Raum sind mehr als 70 nachhaltige Publikumsfonds mit einem Volumen von über 2,4 Milliarden Euro zum Handel zugelassen. Zusammen mit nachhaltigen Spezialfonds, die für einen oder mehrere institutionelle Anleger aufgelegt werden, erreichen nachhaltige Fonds laut Recherchen des Instituts für Markt Umwelt Gesellschaft (imug) ein Gesamtvolumen von 5,9 Milliarden Euro. In Europa belief sich das Volumen der nachhaltigen Publikums- und Spezialfonds Ende 2002 auf 51 Milliarden Euro. Verglichen mit 2001 ist dies ein Wachstum von knapp acht Prozent in einem äußerst schwachen Marktumfeld (1). Je populärer nachhaltige Anlageformen werden, desto größer wird auch das Interesse an den Verfahren, die hinter der Titelauswahl für die jeweiligen Fonds stehen.

Die Auswahl nach ökologischen und sozialen Aspekten wird in der Regel von unabhängigen Research-Agenturen durchgeführt. Heute existieren in Europa etwa zwanzig dieser spezialisierten Organisationen. Renommierte Akteure sind EIRiS, imug, Innovest, oekom research sowie das SiRiNetzwerk (2). Doch auch Banken und Vermögensverwaltungen nehmen eine wichtige Rolle ein. Anders als die spezialisierten Research-Agenturen sind diese in der Regel auch im Management der Anlagen tätig und lassen so den Markt recht heterogen erscheinen. Zu nennen sind hier vor allem die konventionellen Schweizer Banken Sarasin, UBS, Züricher Kantonalbank und die auf Nachhaltigkeit ausgerichtete Vermögensverwaltung $S A M$.
Dow Jones bzw. FTSE aufgelegt worden. Weiterhin haben Ethibel, der belgische SiRi-Partner, und vigeo eigene Indizes entwickelt.

Aufgrund ihrer mehriährigen Erfahrung in der Gestaltung von Nachhaltigkeitsportfolios und nachhaltigen Finanzprodukten sehen sich viele RatingAgenturen in der Lage, Investoren auch hinsichtlich ihrer übergeordneten Nachhaltigkeitsstrategie zu beraten. Das SiRi-Netzwerk und oekom research etwa bieten ihren Finanzkunden solche Dienstleistungen an. In Europa noch wenig verbreitet sind so genannte Engagement Services. Hier zeigen Research-Anbieter ihren Finanzkunden Wege für eine aktive Einflussnahme auf die Umwelt- und Sozialpolitik von Unternehmen auf und stehen ihnen in konkreten Fällen auch beratend zur Seite. CoreRating hat als einziger Anbieter diese Dienstleistung in seiner Selbstdarstellung aufgeführt. Auffällig ist, dass sich fast alle Akteure des europäischen Marktes auf die Analyse von Unternehmen beschränken. Nur die Bank Sarasin, oekom research und die Züricher Kantonalbank bewerten auch Länder und geben ihren Kunden damit die Möglichkeit, Portfolios mit Staatsanleihen, die nach Nachhaltigkeitskriterien ausgewählt sind, zu bestïcken. Während die Research- und RatingAgenturen ihre Produkte Externen zum Kauf anbieten, verbleibt das Research von Sarasin und SAM vorwiegend im eigenen Haus und wird für die Entwicklung nachhaltiger Finanzprodukte verwendet.

\section{Komplexere Analysesysteme}

Auch die verschiedenen Analysesysteme der Rating-Agenturen haben eine Wandlung vollzogen. Während sich die anfänglichen Systeme noch stark auf Aspekte des Umweltmanagements konzentrierten, zeichnen sich ihre weiterentwickelten Versionen durch Differenzierung und Komplexität aus. Kaum eine der Research-Organisationen verzichtet mehr auf soziale Kriterien und auf eine branchenspezifische Untersuchung der Produkte und Dienstleistungen eines Unternehmens. Die Kriterienbreite und -tiefe variiert noch immer, doch die verschiedenen Analyse-Ansätze lassen eine Annäherung erkennen. Voneinander abweichende Bewertungsergebnisse der Agenturen sind zum einen auf unterschiedliche Kriterienkataloge zurückzuführen. Zum anderen sind sie Folge einer differierenden Auffassung des Prinzips Nachhaltigkeit, die sich in der Gewichtung der Kriterien und der Ausformulierung der angewendeten Indikatoren äußert. 


\section{Auf dem Weg zu Qualitätsstandards}

Hinsichtlich der Glaubwürdigkeit sozial-ökologischer Bewertungen stehen neben den untersuchten Kriterien inzwischen auch andere Aspekte im Vordergrund: Jeder Interessierte sollte in der Lage sein, das Bewertungsergebnis eines Unternehmens nachvollziehen zu können (3). Zu diesem Zweck ist die Rating-Systematik öffentlich zugänglich zu machen. Darüber hinaus müssen die Bewertungen Stringenz aufweisen. Um dies zu gewährleisten, hat die Research-Agentur festgelegten Qualitätsstandards zu folgen.

Dieses Thema wurde bereits auf europäischer Ebene aufgegriffen. Derzeit arbeitet eine Initiative verschiedener Rating-Agenturen an einem Entwurf zu freiwilligen Qualitätsstandards für Anbieter von Nachhaltigkeits-Ratings. Dabei konzentriert sich ihre Arbeit auf den Ratingprozess und nicht auf eine Vereinheitlichung von Kriterien, Indikatoren oder Methodiken. Dieselbe Richtung hat auch die Rating-Agentur oekom research eingeschlagen und bereits im Februar diesen Jahres einen solchen Qualitätsstandard veröffentlicht. Darin legt sie allgemeine Pflichten der Research-Agentur fest, die sich auf Faktoren wie Unabhängigkeit, Qualifikation der Analysten oder Transparenz der Ergebnisse beziehen. Weiterhin definiert sie Grundsätze für die Erhebung und Beurteilung von Daten und Informationen und formuliert allgemeine Pflichten der Analysten wie Unparteilichkeit, Gewissenhaftigkeit oder Verschwiegenheit (4).

Neben Transparenz und Stringenz der Analysen ist weiterhin eine institutionelle Trennung der sozialökologischen Bewertung und der Finanzanalyse vonnöten. Denn positive Ergebnisse der Finanzanalyse können den jeweiligen Analysten im sozialen und ökologischen Bereich beeinflussen. Andere Geschäftszweige des Research-Anbieters dürfen die Objektivität der Analysten nicht beeinträchtigen. Werden die Agenturen etwa für Beratungsleistun-gen von Unternehmen bezahlt, die auch von ihr untersucht werden, so ist dies kritisch zu beurteilen. Dasselbe Argument gilt für die Eigentïmerstruktur einer Rating-Agentur. Sie muss neutral und stark gestreut sein, um die Unabhängigkeit und Objektivität der Analysen zu garantieren (5).

\section{- Bleibendes Auswahlproblem für Investoren}

Trotz der Standardisierungstendenzen, die etwa in der Erarbeitung von Qualitätsstandards zu erkennen sind, bleibt das Nachhaltigkeits-Rating ein wertebasiertes und damit subjektives Analysteninstrument. Die Ansichten darüber, was nachhaltig ist und was nicht, können von Investor zu Investor variieren. Verbindliche Leitlinien gibt es nicht. Wer nachhaltig anlegen möchte, sollte sich zunächst Klarheit darüber verschaffen, was er unter Nachhaltigkeit versteht und welche Aspekte dabei für ihn wichtig sind. Diese Überlegungen können in Zusammenarbeit mit einer RatingAgentur erfolgen.

Wer die Dienstleistungen einer Rating-Agentur in Anspruch nehmen möchte, sollte prüfen, ob seine Präferenzen von ihr umgesetzt werden können. Dies gilt sowohl für die zum Einsatz kommenden Kriterien wie auch für das gesamte Leistungspaket der Agentur. Sie sollte maßgeschneiderte Lösungen und keine Dienste von der Stange liefern. Wichtig für die Wahl der passenden Rating-Agentur sind vor allem die zuvor genannten Punkte Vollständigkeit des Kriterienkataloges, Transparenz der Methodik, Trennung von Finanz- und Umwelt- bzw. Sozialanalyse sowie Unabhängigkeit. Für die Ausgestaltung nachhaltiger Finanzprodukte sind die Urteile einer Rating-Agentur von maßgeblichem Einfluss. Aus diesem Grund sollte die Agentur für die Realisierung der Nachhaltigkeitsstrategie selbst zum Untersuchungsobjekt werden.

\section{Anmerkungen}

(1) Vgl. Cerulli Associates: The Cerulli Edge Global Edition, Socially responsible Investing Issue, November 2002, S. 2. (2) Mitglieder von SiRi sind: Avanzi, CaringCompany Etikanalytikera, Centre Info, Dutch Sustainability Research, Fundación Ecologia y Desarollo, KLD, Michael Jantzi Research Associates, Pension \& Investment Research Consultants, scoris, SIRIS, Stock at Stake (Ethibel).

(3) Vgl. auch Lübke, Volkmar: Qualitätsstandards sichern. Wer bewertet die Unternehmensbewerter? In: Ökologisches Wirtschaften, Nr. 6/2001, S.4.

(4) Der Qualitätsstandard von oekom research kann unter www.oekom-research.com eingesehen werden.

(5) Vgl. van den Brink, Timo: Guide Screening and Rating Sustainability. Triple P Performance Centre, Amsterdam 2002, S. 15f. Download unter www.triple-p.org/guide.pdf

\section{Die Autorin}

Marnie Bammert ist Manager Corporate Communications bei oekom research.

Kontakt: oekom research AG, Goethestr. 28, 80336 München, Tel. 089-544184-64,

E-Mail: bammert@oekom-research.com
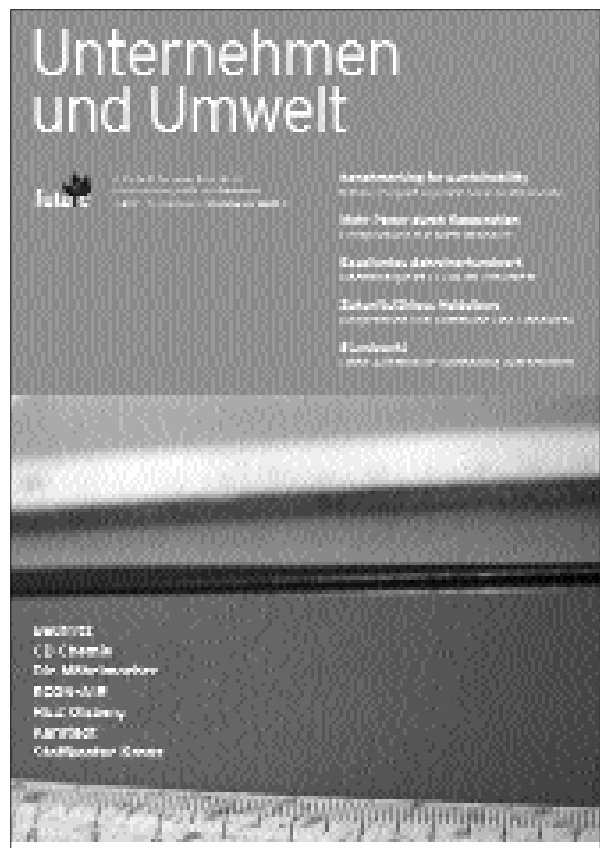

\section{Unternehmen und Umwelt}

.. ist die Zeitschrift für umweltorientierte Unternehmenspolitik von future e.V.

bietet Fachinformationen zum Umweltschutz und Best-Practice-Beispiele aus Unternehmen.

bietet viermal im Jahr Unternehmensbeispiele, Literatur- und Veranstaltungshinweise.

Mit einem Schwerpunktthema in jedem Heft. Zum Beispiel:

3-4/02: Benchmarking for Sustainability 2/02: Ökomarketing

1/02: Nachhaltigkeit und Gewerbebau 4/01: Nachhaltigkeit im Handwerk

3/01: Klimaschutz

2/01: Nachhaltiges Investment

1/01: Grüne Bauwirtschaft

4/00: Kooperative Textilwirtschaft

3/00: Integrierte Produktpolitik

2100: Soft Facts im Umweltschutz

Bestellen Sie ihr kostenloses

Probe-Abo im Internet unter

ww w. future-ev.de

future e.V. Büro Bochum

Am Varenholt 123

44797 Bochum

Fon: 0234/9799513

Fax: 0234/9799514

bochum@future-ev.de

www.future-ev.de 
(c) 20I0 Authors; licensee IÖW and oekom verlag. This is an article distributed under the terms of the Creative Commons Attribution Non-Commercial No Derivates License (http://creativecommons.org/licenses/by-nc-nd/3.o/), which permits unrestricted use, distribution, and reproduction in any medium, provided the original work is properly cited. 